\title{
SARS-CoV-2 transmission dynamics should inform policy
}

\author{
Muge Cevik ${ }^{1}$, Julia L. Marcus ${ }^{2}$, Caroline Buckee ${ }^{3}$, Tara C Smith ${ }^{4}$
}

${ }^{1}$ Division of Infection and Global Health Research, School of Medicine, University of St Andrews, UK

${ }^{2}$ Department of Population Medicine, Harvard Medical School and Harvard Pilgrim Health Care Institute, USA

${ }^{3}$ Center for Communicable Disease Dynamics, Harvard TH Chan School of Public Health, USA

${ }^{4}$ College of Public Health, Kent State University, Kent, USA

\section{Author of correspondence:}

Name: Dr Muge Cevik

Address: Division of Infection and Global Health Research, School of Medicine, University of St Andrews, Fife, KY16 9TF

Telephone number: +447732800814

Email address:mc349@st-andrews.ac.uk

Summary: We argue that SARS-CoV-2 transmission dynamics should inform policy decisions about mitigation strategies for targeted interventions according to the needs of the society

by directing attention to the settings, activities and socioeconomic factors

associated with the highest risks of transmission.

(C) The Author(s) 2020. Published by Oxford University Press for the Infectious Diseases Society of America. All rights reserved. For permissions, e-mail: journals.permissions@oup.com. 


\begin{abstract}
:
It is generally agreed that striking a balance between resuming economic and social activities and keeping the effective reproductive number (R0) below 1 using non-pharmaceutical interventions is an important goal until and even after effective vaccines become available. Therefore, the need remains to understand how the virus is transmitted in order to identify high-risk environments and activities that disproportionately contribute to its spread so that effective preventative measures could be put in place. Contact tracing and household studies in particular provide robust evidence about the parameters of transmission. In this viewpoint, we discuss the available evidence from large-scale, well-conducted contact tracing studies from across the world and argue that SARS-CoV-2 transmission dynamics should inform policy decisions about mitigation strategies for targeted interventions according to the needs of the society by directing attention to the settings, activities and socioeconomic factors associated with the highest risks of transmission.
\end{abstract}

Key words: COVID-19, coronavirus, SARS-CoV-2, novel coronavirus, transmission 


\section{Introduction:}

Since coronavirus disease 2019 (COVID-19) was first described in December 2019, we have witnessed widespread implementation of local and national restrictions in many areas of the world, and social, health and economic devastation due to direct and indirect impact of the pandemic. It is generally agreed that striking a balance between resuming economic and social activities and keeping the effective reproductive number (R0) below 1 using non-pharmaceutical interventions is an important goal until and even after effective vaccines become available. Achieving this balance requires an understanding of how the virus is spread. There is also a need to identify the structural factors that contribute to transmission, a particular concern considering the already stark health disparities driven by socioeconomic and racial/ethnic inequities in our societies.

An understanding of SARS-CoV-2 transmission dynamics can inform policy decisions by directing attention to the settings and activities that confer the highest risk of transmission and understanding of the intersection between poverty, household crowding, and COVID-19. This understanding will allow policymakers and public health practitioners to shape the best strategy, preventative measures and inform the public about transmission risk. Epidemiological investigations including contact tracing studies and outbreak investigations conducted so far across the world already provide crucial information about the probability of infection in close contacts and various environments. We argue that health authorities should use the large-scale, well-conducted contact tracing studies and observations from across the world to date in their risk assessment and mitigation strategies. This article summarizes current knowledge about transmission dynamics and discusses recommendations that could prevent infections by focusing on factors associated with risk of transmission. 


\section{Factors influencing transmission dynamics}

Emerging data suggests that risk of transmission depends on several factors, including contact pattern, host-related infectivity/susceptibility pattern, environment and socioeconomic factors (Figure 1). We will discuss the emerging evidence relating to each of these aspects of transmission.

\section{1- Contact pattern}

Contact tracing studies provide early evidence that sustained close contact drives the majority of infections and clusters. For instance, living with the case, family/friend gatherings, dining, or travelling on public transport were found to have a higher risk for transmission than market shopping or brief (<10 mins) community encounters [1-3]. While people are more likely to recall and disclose close and household contacts, and it is easier for tracers to identify the source, household studies provide important information about the contact patterns and activities associated with higher attack rates. Close contacts with the highest risk of transmission are typically friends, household members, and extended family, with a secondary attack rate that ranges from 4 to $35 \%[1,4-8]$. In the same household, higher attack rates are observed among spouses compared to the rest of the household [8]. In a systematic review including five studies based on relationship demonstrated that household SAR to spouses $(43,4 \%$; $95 \%$ CI: $27,1 \%-59,6 \%)$ was significantly higher than to other relationships (18,3\%, 95\% CI: $10,4 \%-26,2 \%)$ [8]. Similar results were observed in the USS Theodora Roosevelt outbreak in which those sharing the same sleeping space had higher risk of being infected [9]. In addition, the attack rate has shown to be higher when the index case is isolated in the same room with the rest of the household or when the household members have daily close contact with the index case $[10,11]$. Transmission is significantly reduced when the index case is isolated away from the family, or preventative measures such as social distancing, hand hygiene, disinfection and use of face masks at home are applied $[10,11]$. In a study of an outbreak in the largest meat processing plant in Germany, while the universal point of potential contact among all cases was workplace, positive rates were statistically significant for a single shared apartment, shared bedroom and associated carpool 
[12]. These findings suggest that sleeping in the same room or sharing the same sleeping space, increased contact frequency constitutes high risk of transmission.

Large clusters have been observed in family, friend, work-colleague gatherings including weddings and birthday parties $[13,14]$. Other examples include gatherings in pubs, church services, and close business meetings [14-17]. These findings suggest that group activities pose a higher risk of transmission. In non-household contact tracing studies, dining together or engaging in group activities such as board games have been found to be high risk for transmission as well [18]. In the same household, frequent daily contact with the index case, and dining in close proximity has been associated with increased attack rates $[10,11]$.

Large, long-term care facilities such as nursing homes and homeless shelters have seen increased rates of infection, in part because of patterns of contact among staff and residents. In nursing home outbreak investigations from the Netherlands, Boston, and London, multiple viral genomes were identified, suggesting multiple introductions to the facility leading to infections among residents [1921]. In an investigation of 17 nursing homes that implemented voluntary staff confinement with residents, including 794 staff members and 1250 residents in France, staff confining themselves to a single facility for a weeklong period was associated with decreased outbreaks in these facilities [22].

These findings emphasise that contact patterns, including the duration of contact, contact frequency, proximity to index case and types of activities influence transmission risk, highlighting the need for tailored prevention strategies for different settings. 


\section{2- Host factors}

Contact tracing and outbreak investigations suggest that many SARS-CoV-2-infected people either do not contribute to onward transmission or have minimal potential to do so [6, 17], and a large number of secondary cases are often caused by a small number of infected patients. While this may also be due to contact pattern and the environmental factors, host factors strongly influence this variation; individual variation in infectiousness is an expected feature of superspreading events.

Timing of the contact with an index case is key in transmission dynamics as it relates to the infectiousness of the index case. In a living systematic review of studies published up to 6 June 2020, we found that viral load peaks early in the disease course, with the highest viral loads observed from symptom onset to day 5, indicating high level of infectiousness during this period [23] (Figure 2). Supporting these findings, transmission events are estimated to occur in a short window, likely a few days prior to and following symptom onset $[4,23]$. For example, a contact tracing study that followed up 2761 contacts of 100 confirmed COVID-19 cases demonstrated that infection risk was higher if the exposure occurred within the first five days after the symptom onset, with no secondary cases documented after this point [4]. This understanding indicates that viral dose plays an important role in transmission dynamics. In contrast, higher viral loads in SARS-CoV-1 and MERS-CoV were identified in the second week after symptom onset, suggesting that patients had viral load peak after hospitalisation [23]. Therefore, early viral load peak also explains efficient community SARS-CoV-2 spread in contrast to SARS-CoV-1 and MERS-CoV during which community spread was put under control; however, nosocomial spread was an important feature of the outbreaks. In contrast during COVID-19, only a small number of hospital-based outbreaks have been reported so far, which may be due to downtrend viral load levels later in disease course [23, 24].

Symptoms and severity of illness appear to influence transmission dynamics as well. People with symptoms appear to have a higher secondary attack rate compared to pre-symptomatic and 
asymptomatic index cases (those who develop no symptoms throughout the illness) [18]. While asymptomatic patients can transmit the virus to others, the findings from nine studies in a systematic review, including studies published up to 3 July 2020, found secondary attack rates of zero to $2.8 \%$, compared with secondary attack rates of $0.7 \%$ to $16.2 \%$ in symptomatic cases in the same studies, suggesting asymptomatiic index cases transmit to fewer secondary cases [18]. Another systematic review that included studies published up to 10 June 2020 similarly found a reduced risk of transmission for asymptomatic versus symptomatic cases $(0.35,95 \%$ CI $0.10,1.27)$ and presymptomatic versus symptomatic cases $(0.63,95 \%$ CI $0.18,2.26)$ [25]. There are also differences in attack rates based on symptom severity. In the Zhang et al. study the secondary attack rate was 3.5\% for those with mild symptoms, $5.7 \%$ for those with moderate symptoms, and $4.5 \%$ for those with severe symptoms (based on CDC China guidelines) [26]. In a contact tracing study, contacts of severe cases were more likely to develop severe infections themselves [4].

Virus transmission is also affected by a number of other host factors, including host defense mechanisms, and age. Current synthesis of the literature demonstrates significantly lower susceptibility to infection for children aged under 10 years compared to adults given the same exposure, and elevated susceptibility to infection in adults aged over 60 years compared to younger or middle-aged adults [27].

\section{3- Environment}

Transmission risk is not one-dimensional and contact patterns also depend on the setting of the encounter. Findings from contact tracing studies in Japan suggest an 18.7-fold higher risk of transmission indoors compared with outdoor environments [28]. These findings are in keeping with our understanding about transmission patterns of respiratory viral infections. While outdoor settings usually have lower risk, prolonged contact in an enclosed setting can lead to increased risk of transmission. Especially when combined with environmental factors such as poor ventilation and crowding this may lead to further increase in attack rates. Epidemiological studies so far support this 
knowledge. SARS-CoV-2 is much more efficiently spread in enclosed and crowded environments. Largest outbreaks from across the world are reported in long term care facilities such as nursing homes, homeless shelters, prisons, and also workplaces including meat-packing plants and factories, where many people spend several hours working together, dining and sharing communal spaces $[12$, 14]. In six London care homes experiencing SARS Cov-2 outbreaks identified a high proportion of residents (39.8\%) and staff (20.9\%) tested positive for SARS-CoV-2 [20]. Among 408 individuals residing at a large homeless shelter in Boston, $36 \%$ of those tested were found to be positive [16]. Although it is much harder to obtain data from incarcerated populations, the largest clusters of cases observed in the USA have all been associated with prisons or jails, suggesting a high attack rate in these institutional settings [29]. Social distancing is the opposite of incarceration, and overcrowding, poor sanitation and ventilation, and inadequate healthcare contribute to the disproportionate rates of infections seen in prisons and jails, which demonstrates the larger pattern of the health disparities in our societies.

\section{4- Socioeconomic factors and racial/ethnic disparities}

Global figures suggest that there is a strong association between socioeconomic deprivation, race/ethnicity and a higher risk of infection and death from COVID-19 [30, 31]. People facing the greatest socioeconomic deprivation experience a higher risk of household and occupational exposure to SARS-CoV-2, and existing poor health leads to more severe outcomes if infected [32]. People with lower-paid and public-facing occupations are often classified as essential workers who must work outside the home and may travel to work on public transport. Indeed, in New York City, higher cumulative infection rates were observed in neighbourhoods that continued to engage in mobility behaviours consistent with commuting for work [33]. These occupations often involve greater social mixing and greater exposure risk due to prolonged working hours, resulting in reduced ability to practice social distancing among low-income families [34]. In addition, households in socioeconomically deprived areas are more likely to be overcrowded, increasing the risk of 
transmission within the household. Black, Hispanic, and other marginalised, racial/ethnic and migrant groups have also been shown to be at greater risk of infection, severe disease, and death from COVID-19 [31, 35-37]. These increased risks are also likely due to socioeconomic conditions that increase risk of transmission, inequitable access to adequate healthcare, and higher rates of comorbidities due to adverse living and working conditions and structural racism. It is not surprising that the largest outbreaks are observed in meat-packing plants, and most commonly exposed occupations include nurses, taxi and bus drivers and factory workers [31]. These disparities also shape the strong geographic heterogeneities observed in the burden of cases and deaths, for example across the USA and the UK $[31,38]$. These findings support the hypothesis that the COVID19 pandemic is strongly shaped by structural inequities that drive household and occupational risks, emphasising the need to tailor effective control and recovery measures for these disadvantaged communities proportionate to their greater needs and vulnerabilities.

\section{5- Large clusters and superspreading events}

Clusters have become a prominent characteristic of SARS-CoV-2, which distinguishes it from seasonal influenza $[14,17]$. This emphasises that large clusters and superspreading events may be the driver of the majority of infections, just as they were for SARS in 2002-2003 [39, 40]. For instance, during the 2003 SARS outbreak, over $70 \%$ of infections were linked to superspreading events in Hong Kong and Singapore [39]. Hallmarks for superspreading events include a combination of factors, typically a highly infectious individual(s) gathered with other individuals in enclosed and crowded environments $[14,17]$. There have been several superspreading events reported so far. For example, an outbreak investigation from China identified that 24 out of 67 passengers were infected during a 50-minute return bus journey, which was linked to an index case who was symptomatic the day before the trip. In contrast, during the event, only six people were infected, all of whom were in close contact with the same index case [41]. In Washington state, a mildly symptomatic index case attended a choir practice (the practice was 2.5 hours), and out of 61 persons, 32 confirmed and 20 
probable secondary COVID-19 cases occurred with an attack rate of 53.3\% to 86.7\%) [42]. While these superspreading events occur, the frequency of these events and whether they are caused by a single index case are unclear. The modelling suggests that several independent introductions might be needed before a COVID-19 outbreak eventually takes off, meaning often these large outbreaks occur when multiple infected persons are introduced to the environment as shown in the nursing home investigation [43]. Other large outbreaks are reported in night clubs, karaoke bars, pubs [14, 17], which may be related to crowding, leading to multiple introductions into the same setting as seen in nursing home investigations. These findings and observations suggest that contact tracing investigations need to be combined with phylogenetic analysis to understand the settings and activities most likely to yield a superspreading event to inform preventative measures.

\section{Recommendations}

Increased risk of transmission in deprived areas and among people in low-paid jobs suggest that poverty and household crowding need to be addressed with interventions that go beyond guidance on social distancing, hand hygiene, and mask use. Previous research suggests that although social distancing during the $2009 \mathrm{H} 1 \mathrm{~N} 1$ swine flu pandemic was effective in reducing infections, this effect was most pronounced in households with greater socioeconomic advantage. Similar findings are emerging for COVID-19, with the ability to practice social distancing strongly differentiated by county and household income [34]. The disproportionate impact of COVID-19 on households living in poverty, and the racial and ethnic disparities observed in many countries, emphasize the need to urgently address these inequities that directly impact health outcomes. This includes social and income protection and support to ensure low paid, non-salaried and zero-hours contract workers can afford to follow isolation and quarantine recommendations, provision of protective equipment for workplaces and community settings, appropriate return-to-work guidelines, and testing and opportunities for isolation outside of the home to protect those still at work. 
Second, knowing which contacts and settings confer the highest risk for transmission can help direct contact tracing and testing efforts to increase the efficiency of mitigation strategies. Early viral load peak in the disease course indicates that preventing onward transmission requires immediate selfisolation with symptom onset, prompt testing and results with a 24-48 hours turnaround time, and robust contact tracing. In many countries, people with symptoms access testing late in the disease course, by which time they may have had multiple contacts while in the most infectious period. While self-isolation with symptoms is crucial, $75 \%$ of those with symptoms and their contacts in the UK reported not fully self-isolating [44]. While presymptomatic transmission likely contributes to a fraction of onward transmission, over half of transmission is caused by those with symptoms, especially in the first few days after symptom onset. These findings suggest that messages should prioritise isolation practice, and policies should include supported isolation and quarantine.

Third, policy makers and health experts can help the public differentiate between lower-risk and higher-risk activities and environments and public health messages could convey a spectrum of risk to the public to support engagement in alternatives for safer interaction, such as in outdoor settings. Without clear public health communication about risk, individuals may fixate on unlikely sources of transmission —outdoor activities - while undervaluing higher-risk settings, such as family and friend gatherings, and indoor settings. Enhancing community awareness about risk can also encourage symptomatic persons and contacts of ill persons to isolate or self-quarantine to prevent ongoing transmission.

Finally, because crowded, indoor spaces and gatherings likely will continue to be the driver of transmission, public health strategies will be needed to mitigate transmission in these settings, such as nursing homes, prisons and jails, shelters, meat-packing plants such as personal protective equipment and routine testing to identify infected individuals early in the disease course. As part of the pandemic response we may need to consider fundamentally redesigning these settings, including improved 
ventilation, just as improved sanitation was a response to cholera. Such strategies should be adopted in settings where large outbreaks and superspreading events have been identified by contact tracing studies.

While modelling studies and computer simulations could contribute to our understanding of transmission dynamics and aero-dynamics of droplets, contact-tracing studies provide real-life transmission dynamics, individual and structural factors associated with SARS-CoV-2 transmission, which are essential to shape our public health plans, mitigate superspreading events, and control the current pandemic. Further understanding of transmission dynamics is also critical to developing policy recommendations for reopening businesses, primary and secondary schools, and universities. 
NOTES

\section{Acknowledgements:}

N/A

\section{Funding:}

JM is supported in part by the U.S. National Institute of Allergy and Infectious Diseases [K01 AI122853].

\section{Conflict of interest:}

MC, CB, TS have nothing to declare. JM has consulted for Kaiser Permanente Northern California on a research grant from Gilead Sciences and 
References:

1. Chen Yi WA, Yi Bo, Ding Keqin, Wang Haibo, Wang Jianmei, Shi Hongbo, Wang Sijia , Xu Analysis of epidemiological characteristics of infections in close contacts with new coronavirus pneumonia in Ningbo

. Chinese Journal of Epidemiology 2020.

2. Chaw L, Koh WC, Jamaludin SA, Naing L, Alikhan MF, Wong J. SARS-CoV-2 transmission in different settings: Analysis of cases and close contacts from the Tablighi cluster in Brunei Darussalam. 2020.

3. Zhang JZ, Zhou P, Han DB, et al. [Investigation on a cluster epidemic of COVID-19 in a supermarket in Liaocheng, Shandong province]. Chung Hua Liu Hsing Ping Hsueh Tsa Chih 2020; 41(0): E055.

4. Cheng H-Y, Jian S-W, Liu D-P, et al. Contact Tracing Assessment of COVID-19 Transmission Dynamics in Taiwan and Risk at Different Exposure Periods Before and After Symptom Onset. JAMA Internal Medicine 2020.

5. Burke RM, Midgley CM, Dratch A, et al. Active Monitoring of Persons Exposed to Patients with Confirmed COVID-19 - United States, January-February 2020. MMWR Morb Mortal Wkly Rep 2020; 69(9): 245-6.

6. $\mathrm{Bi} \mathrm{Q}, \mathrm{Wu} \mathrm{Y}, \mathrm{Mei} \mathrm{S}$, et al. Epidemiology and transmission of COVID-19 in 391 cases and 1286 of their close contacts in Shenzhen, China: a retrospective cohort study. The Lancet Infectious Diseases.

7. Lopez Bernal J, Panagiotopoulos N, Byers C, et al. Transmission dynamics of COVID-19 in household and community settings in the United Kingdom. medRxiv 2020: 2020.08.19.20177188.

8. Madewell ZJ, Yang Y, Longini IM, Halloran ME, Dean NE. Household transmission of SARS-CoV-2: a systematic review and meta-analysis of secondary attack rate. medRxiv 2020: 2020.07.29.20164590.

9. Payne DC, Smith-Jeffcoat SE, Nowak G, et al. SARS-CoV-2 Infections and Serologic Responses from a Sample of U.S. Navy Service Members - USS Theodore Roosevelt, April 2020. MMWR Morb Mortal Wkly Rep 2020; 69(23): 714-21.

10. Wang $Y$, Tian $H$, Zhang $L$, et al. Reduction of secondary transmission of SARS-CoV2 in households by face mask use, disinfection and social distancing: a cohort study in Beijing, China. BMJ Global Health 2020; 5(5): e002794.

11. Böhmer MM, Buchholz U, Corman VM, et al. Investigation of a COVID-19 outbreak in Germany resulting from a single travel-associated primary case: a case series. The Lancet Infectious Diseases.

12. Guenther T, Czech-Sioli, Manja, Indenbirken, Daniela, Robitailles, Alexis, Tenhaken, Peter, Exner, Martin, Ottinger, Matthias, Fischer, Nicole, Grundhoff, Adam, Brinkmann, Melanie, . Investigation of a superspreading event preceding the largest meat processing plant-related SARS-Coronavirus 2 outbreak in Germany. SSRN 2020.

13. Ghinai I, Woods S, Ritger KA, et al. Community Transmission of SARS-CoV-2 at Two Family Gatherings - Chicago, Illinois, February-March 2020. MMWR Morb Mortal Wkly Rep 2020; 69(15): 446-50.

14. Leclerc QJ FN, Knight LE. What settings have been linked to SARS-CoV-2 transmission clusters? [version 1; peer review: 1 approved with reservations]. Wellcome Open Res 2020; 5:83

15. Arons MM, Hatfield KM, Reddy SC, et al. Presymptomatic SARS-CoV-2 Infections and Transmission in a Skilled Nursing Facility. New England Journal of Medicine 2020.

16. Baggett TP, Keyes H, Sporn N, Gaeta JM. Prevalence of SARS-CoV-2 Infection in Residents of a Large Homeless Shelter in Boston. JAMA 2020. 
17. Dillon Adam PW, Jessica Wong et al. Clustering and superspreading potential of severe acute respiratory syndrome coronavirus 2 (SARS-CoV-2) infections in Hong Kong

. PREPRINT (Version 1) available at Research Square 2020.

18. Qiu X, Nergiz AI, Maraolo AE, Bogoch II, Low N, Cevik M. Defining the role of asymptomatic SARS-CoV-2 transmission: a living systematic review. medRxiv 2020: 2020.09.01.20135194.

19. Helene ACM Voeten RSS, Marjolein Damen et al. . Unravelling the modes of transmission of SARS-CoV-2 during a nursing home outbreak: looking beyond the church super-spread event. Research Square 2020.

20. Ladhani SN, Chow JY, Janarthanan R, et al. Investigation of SARS-CoV-2 outbreaks in six care homes in London, April 2020. EClinicalMedicine.

21. Lemieux J, Siddle KJ, Shaw BM, et al. Phylogenetic analysis of SARS-CoV-2 in the Boston area highlights the role of recurrent importation and superspreading events. medRxiv 2020: 2020.08.23.20178236.

22. Belmin J, Um-Din N, Donadio C, et al. Coronavirus Disease 2019 Outcomes in French Nursing Homes That Implemented Staff Confinement With Residents. JAMA Network Open 2020; 3(8): e2017533-e.

23. Cevik M, Tate M, Lloyd O, Maraolo AE, Schafers J, Ho A. SARS-CoV-2, SARS-CoV1 and MERS-CoV viral load dynamics, duration of viral shedding and infectiousness: a living systematic review and meta-analysis. medRxiv 2020: 2020.07.25.20162107.

24. Cevik M, Bamford CGG, Ho A. COVID-19 pandemic-a focused review for clinicians. Clin Microbiol Infect 2020; 26(7): 842-7.

25. Buitrago-Garcia DC, Egli-Gany D, Counotte MJ, et al. The role of asymptomatic SARS-CoV-2 infections: rapid living systematic review and meta-analysis. medRxiv 2020: 2020.04.25.20079103.

26. Zhang W, Cheng W, Luo L, et al. Secondary Transmission of Coronavirus Disease from Presymptomatic Persons, China. Emerg Infect Dis 2020; 26(8).

27. Goldstein E, Lipsitch M, Cevik M. On the effect of age on the transmission of SARSCoV-2 in households, schools and the community. medRxiv 2020:

2020.07.19.20157362.

28. Nishiura $\mathrm{H}$, Oshitani $\mathrm{H}$, Kobayashi $\mathrm{T}$, et al. Closed environments facilitate secondary transmission of coronavirus disease 2019 (COVID-19). medRxiv 2020: 2020.02.28.20029272.

29. Franco-Paredes C, Jankousky K, Schultz J, et al. COVID-19 in jails and prisons: A neglected infection in a marginalized population. PLOS Neglected Tropical Diseases 2020; 14(6): e0008409.

30. Koma W, Artiga, S., Neuman, T., Claxton, G.,Rae,M.,Kates,J.,Michaud, J. . LowIncome and Communities of Color at Higher Risk of Serious Illness if Infected with Coronavirus

. Available at: https://www.kff.org/coronavirus-covid-19/issue-brief/low-income-andcommunities-of-color-at-higher-risk-of-serious-illness-if-infected-with-coronavirus/. Accessed July 21.

31. Statistics OfN. Deaths involving COVID-19 by local area and socioeconomic deprivation: deaths occurring between 1 March and 31 May 2020

\section{Available at:}

https://www.ons.gov.uk/peoplepopulationandcommunity/birthsdeathsandmarriages/d eaths/bulletins/deathsinvolvingcovid19bylocalareasanddeprivation/deathsoccurringbe tween1marchand31may2020. Accessed 2020.

32. Rose TC, Mason K, Pennington A, et al. Inequalities in COVID19 mortality related to ethnicity and socioeconomic deprivation. medRxiv 2020: 2020.04.25.20079491.

33. Kissler SM, Nishant Kishore, Malavika Prabhu, Dena Goffman, Yaakov Beilin, et al. . Reductions in commuting mobility predict geographic differences in SARS-CoV-2 prevalence in New York City

. Harvard Library 2020. 
34. Weill JA, Stigler M, Deschenes O, Springborn MR. Social distancing responses to COVID-19 emergency declarations strongly differentiated by income. Proceedings of the National Academy of Sciences 2020; 117(33): 19658.

35. Rodriguez-Diaz CE, Guilamo-Ramos V, Mena L, et al. Risk for COVID-19 infection and death among Latinos in the United States: Examining heterogeneity in transmission dynamics. Annals of Epidemiology 2020.

36. Millett GA, Jones AT, Benkeser D, et al. Assessing differential impacts of COVID-19 on black communities. Annals of Epidemiology 2020; 47: 37-44.

37. Okonkwo NE, Aguwa UT, Jang M, et al. COVID-19 and the US response: accelerating health inequities. BMJ Evidence-Based Medicine 2020: bmjebm-2020111426.

38. Chin T, Kahn R, Li R, et al. US-county level variation in intersecting individual, household and community characteristics relevant to COVID-19 and planning an equitable response: a cross-sectional analysis. BMJ Open 2020; 10(9): e039886.

39. Li Y, Yu ITS, Xu P, et al. Predicting Super Spreading Events during the 2003 Severe Acute Respiratory Syndrome Epidemics in Hong Kong and Singapore. American Journal of Epidemiology 2004; 160(8): 719-28.

40. Shen Z, Ning F, Zhou W, et al. Superspreading SARS events, Beijing, 2003. Emerg Infect Dis 2004; 10(2): 256-60.

41. Shen Y LC, Dong H, Wang Z, Martinez L, Sun Z, et al. . Airborne transmission of COVID-19: epidemiologic evidence from two outbreak investigations. . PREPRINT 2020.

42. Hamner L, Dubbel P, Capron I, et al. High SARS-CoV-2 Attack Rate Following Exposure at a Choir Practice - Skagit County, Washington, March 2020. MMWR Morb Mortal Wkly Rep 2020; 69(19): 606-10.

43. Kucharski AJ, Russell TW, Diamond C, et al. Early dynamics of transmission and control of COVID-19: a mathematical modelling study. The Lancet Infectious Diseases 2020; 20(5): 553-8.

44. Smith LE, Amlôt R, Lambert H, et al. Factors associated with adherence to selfisolation and lockdown measures in the UK: a cross-sectional survey. Public Health 2020; 187: 41-52. 


\section{Figure legends}

\section{Figure 1: Factors influencing transmission dynamics}

Transmission depends on several factors, including contact pattern (duration of contact, gathering, proximity, activity), environment (outdoor, indoor, ventilation), host-related infectivity/susceptibility pattern (i.e. viral load in relation to disease course, severity of illness, age) and socioeconomic factors (i.e. crowded housing, job insecurity, poverty). Virus infectivity and differences between other viruses, and host immune factors are not discussed in this review. (This figure is created by the authors based on available literature about SARS-CoV-2 transmission dynamics)

\section{Figure 2: SARS-CoV-2 viral load dynamics and period of infectiousness}

Incubation period (time from exposure to symptom onset) 6 days (2-21 days), peak viral load levels documented from day 0 (symptom onset) to day5, infectious period starts before symptom onset up to 10 days (this may be extended in patients with severe illness), RNA shedding continues for a prolonged period of time but culturable virus has been identified up to day 9 of illness. (This figure is created by the authors on Biorender based on available literature about SARS-CoV-2 viral load dynamics) 
Figure 1

Indoor/Outdoo

Ventilation

Long term care facilities

Proximity to index case

Time of contact

Duration of exposure

Contact frequency

Activity

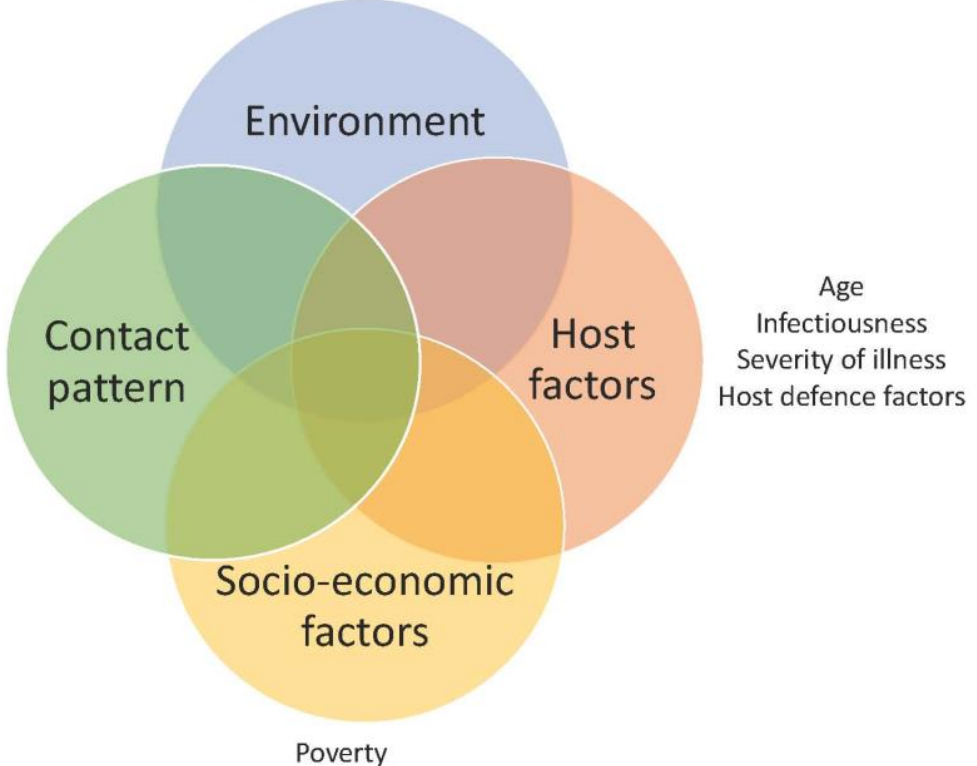

Poverty

rolonged working hours

Household crowding 
SARS-CoV-2 viral load and period of infectiousness Cevik M et al. https://doi.org/10.1101/2020.07.25.20162107

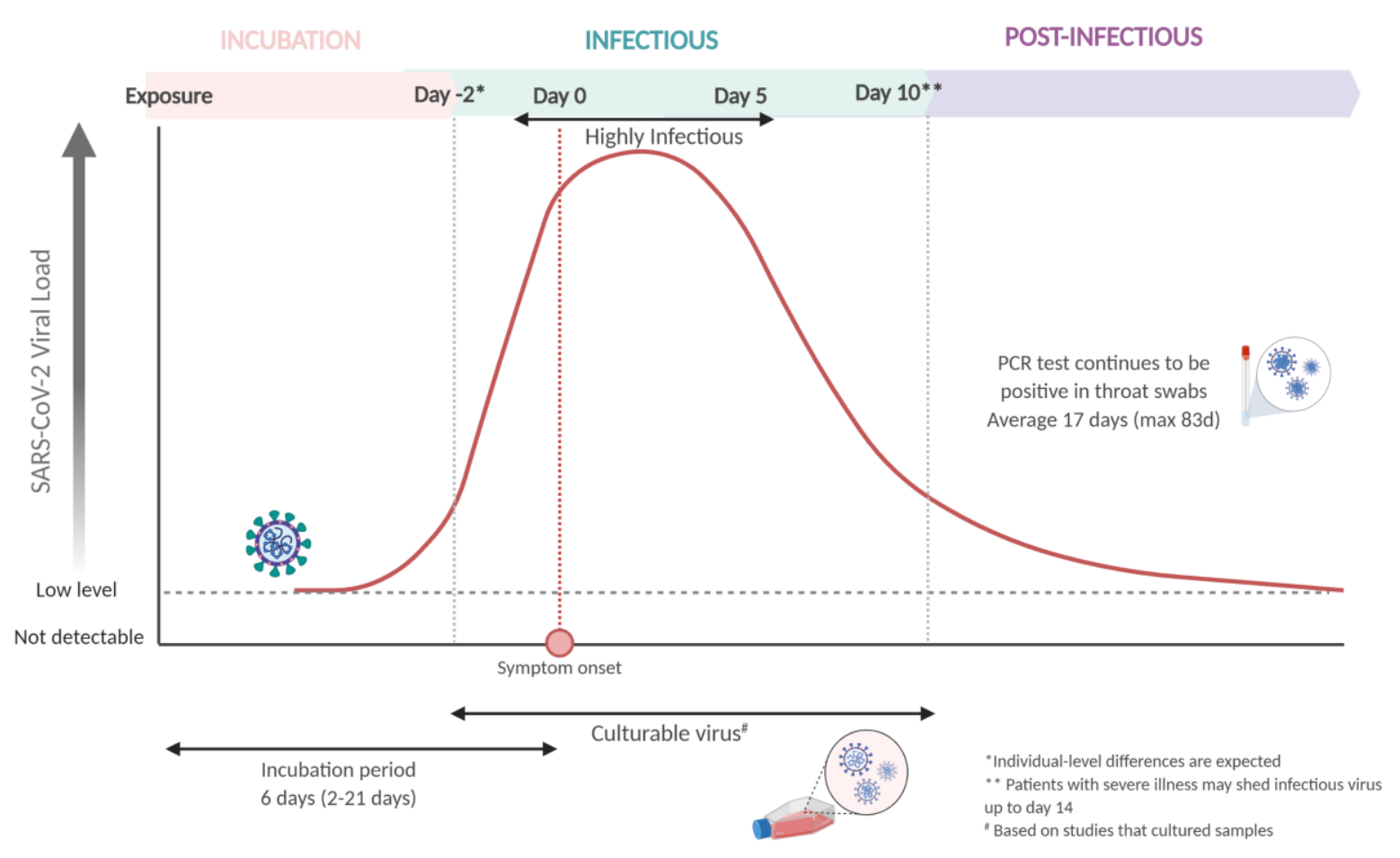

